\title{
Cold agglutinins - a common clinical or laboratory problem. Clinical case
}

\begin{abstract}
Introduction: The examination of the blood picture is an initial stage in the diagnostic process, both in anaemic and inflammatory conditions. Agglutination of erythrocytes and platelets, as well as hemolysis, can lead to "wrong" values when automatically enumerated with haematology analysers.
\end{abstract}

Purpose: We present a case of in vitro erythrocyte agglutination caused by cold agglutinins as a result of viral pneumonia with the etiologic agent influenza B.

Material and methods: This is a man entering a pulmonary ward with pneumonia. The examined blood picture shows abnormal values of the red blood row. The analysis was performed on a Sysmex XN1000 haematology analyzer. The flags and cell cytograms of the apparatus were examined. The morphology of the cellular elements (erythrocytes, leukocytes-DCL and platelets) of stained blood smear was examined microscopically. A saline test was applied to differentiate the in vitro agglutination. We determined the biochemical markers for hemolysis (free haemoglobin in plasma, LDH, Haptoglobin of Olympus AU 640 analyser), the titer of cold agglutinins. Virological serological studies have been appointed.

Results: CPC values were monitored from admission to patient discharge and at the end of the first month. The biochemical markers for hemolysis are poorly expressed. The saline test and the erythrocyte morphology give direction to in vitro agglutination. A virological examination found antibodies against influenza type $B$ and Coxiella burnetii ( $Q$ fever).

Conclusion: A good knowledge of artificially occurring in vitro phenomena resulting from erythrocyte agglutination can avoid a number of misunderstandings or misinterpretations.

Keywords: cold agglutinins, cells cytograms, RBC, erythrocyte indices, blood smear
Volume 5 Issue 2 - 2020

\author{
Mariana Yordanova ${ }^{1,2}$ \\ 'Military Medical Academy, Multiprofile Hospital for Active \\ Treatment, Bulgaria \\ ${ }^{2}$ Department of General Medicine and Clinical Laboratory, \\ Medical University of Varna "Prof. Dr. Paraskev Stoyanov", \\ Bulgaria
}
Correspondence: Mariana Yordanova, General Medicine and Clinical Laboratory, Medical University of Varna "Prof. Paraskev Stoyanov", Varna, Bulgaria, Tel 359882799904, Emailm.jordaova.doc@abv.bg

Received: February 23, 2020 | Published: March 04, 2020

\section{Introduction}

The in vitro phenomenon leading to agglutination of erythrocytes, platelets and leukocytes, during their counting in the analyser is the cause of analytical "errors" requiring attention and knowledge. The main cause of such agglutination is the presence of antibodies. All individuals have low concentrations of circulating antibodies (Ab) directed against blood cells, but they do not cause any clinical or laboratory effect. In certain external circumstances, such as low temperature, anticoagulants in the blood sample, they are activated and lead to some in vitro laboratory phenomena, with or without clinical manifestation.

Anti-erythrocyte cold antibodies at higher concentrations cause erythrocytes to clot (and agglutinate) and/or lysis. They are activated at low temperatures below $30^{\circ} \mathrm{C} .{ }^{1}$ Cold agglutinins were first described by Landsteiner in $1903 .^{2}$ They target the polysaccharide region of glycoproteins on the surface of red blood cells (usually I antigen, i antigen, and Pr antigens). ${ }^{3}$ Mostly IgM dominated and rarely IgA or IgG class. Autoerythroantibodies, ${ }^{4}$ which are typically found serum in most people, are only active at temperatures up to $10^{\circ}-15^{\circ} \mathrm{C}$. and have low activity and titer (1:32). The temperature range of their operation may vary too wide. However, to achieve a hemolytic effect, Ab must be either in large quantity or, if in low concentration, the optimal blood temperature for this reaction should be about $4^{\circ} \mathrm{C}$. The resulting antigen-antibody complex strongly activates the classical complement cascade, leading to the destruction of RBCs in the reticuloendothelial system, intravascular and extravascular. Cold agglutinin disease $(\mathrm{CAD})$ is a rare autoimmune disease leading to hemolytic anaemia. It is traditionally classified as primary (idiopathic type) and secondary, accompanying inflammatory and lymphoproliferative disorders. ${ }^{5}$ Polyclonal or post-infectious cold agglutinins (CA) are mainly found in viral and parasitic infections such as mycoplasma pneumonia, influenza A and B, infectious mononucleosis, HIV, malaria and more. ${ }^{6}$ While Monoclonal cold IgM class antibodies are synthesised in lymphomas. $^{2}$

CAs that occur in the course of/or after infection usually do not lead to serious complaints, have no significant clinical manifestation, require no treatment, and are limited to about a few weeks. But they can cause an in vitro phenomenon in the blood cell count. Postinfectious CAD usually occurs at a younger age than the monoclonal variant. Extensive research is required to clarify the root cause. The set of indicators should include reticulocyte count, serum lactate dehydrogenase, serum bilirubin, free plasma haemoglobin, haptoglobin, urine analysis (haemoglobin and urobilinogen), direct and indirect Coombs test. ${ }^{2}$

\section{Case report}

We present the case of a 36-year-old man entering the pulmonology department for complaints of chills and fever up to 
$38^{\circ} \mathrm{C}$, profuse sweating, progressive fatigue, dry and irritating cough The complaints date back to about 10 days. It was initially reviewed by the GP. An X-ray was made where data on right-sided pneumonia were established. He was appointed AB therapy (Avirone Rapid), but the treatment has no effect. For the last 2-3 days, the patient has shortness of breath, easy fatigue with little physical effort. Due to his deterioration, he was admitted to the ward.The blood test shows the following results given in Table 1 .

Table I Peripheral blood count of the patient at the time of admission to hospital, determined on a Sysmex XNI000 hematology analyser

\begin{tabular}{llllll}
\hline Parameters & I day & 2day & 3day & $\begin{array}{l}\text { after } 3 \\
\text { weeks }\end{array}$ & Unit \\
\hline HGB & 145.0 & 134.0 & 128.0 & 139.0 & $\mathrm{~g} / \mathrm{L}$ \\
RBC & 1.01 & 0.65 & 0.92 & 4.94 & $\times 10^{\Lambda} \mathrm{I} / \mathrm{L}$ \\
HCT & 0.093 & 0.061 & 0.092 & 0.429 & $\mathrm{~L} / \mathrm{L}$ \\
WBC & 13.33 & 8.7 & 10.13 & 7.55 & $\times 10^{\Lambda} 9 / \mathrm{L}$ \\
MCV & 92.1 & 93.8 & 100.0 & 86.8 & $\mathrm{fL}$ \\
MCH & 143.6 & 206.2 & 139.1 & 28.1 & $\mathrm{Pg}$ \\
MCHC & 1559.0 & 2197.0 & 1391.0 & 324.0 & $\mathrm{~g} / \mathrm{L}$ \\
RDW & 15.3 & ---- & ---- & 13.2 & $\%$ \\
PIT & 420.0 & 369.0 & 368.0 & 205.0 & $\times 10^{\Lambda} 9 / \mathrm{L}$ \\
\hline
\end{tabular}

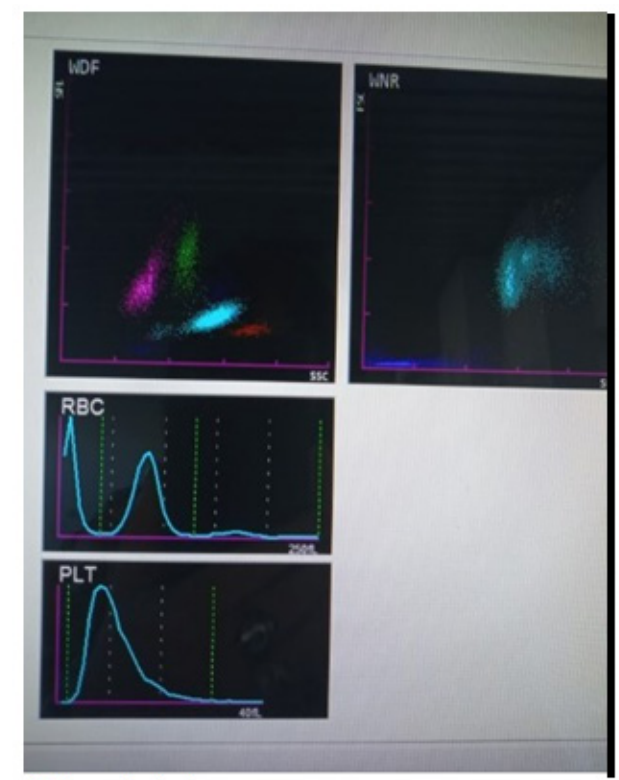

A) On admission
The resulting low red blood cells and hematocrit for the corresponding haemoglobin (at hospitalization $145.0 \mathrm{~g} / \mathrm{L}$ ), combined with high erythrocyte index values, drew attention to a more thorough study of the histograms and flags given as analyser counts. Within 3-4 days there is a decrease in haemoglobin values to low-grade anaemia.

Erythrocyte cytograms (scartograms) can be used for rapid orientation about changes in cell morphology. Specific cytograms for reticulocytosis, cold agglutinins in the sample, spherocytes, schizocytes, target cells, hematologic conditions, etc. are described. ${ }^{7,8}$ Typically, the distribution curve of the RBC is symmetrical in the form of a bell (Gaussian distribution). The peak of the curve falls within the normal range of the $\mathrm{MCV}$, which is $80-100 \mathrm{fl}$ (range between $25 \mathrm{fl}$ and $360 \mathrm{fl}){ }^{8}$ When the RBC population is homogeneous, it gives a narrow distribution curve.

In the presence of cold agglutinins, the curves may vary in time and temperature-dependent forms. Their appearance is similar to $\mathrm{U}$ (Figure 1). The high titer of cold agglutinins leads to agglutination of erythrocytes and interferes with their sizing, enumeration and calculation of RDW. ${ }^{8}$ Doubled or triplet red blood cells are counted as 1 cell, and larger groups of cells are not counted as RBCs, resulting in a change in number and volume. This changes all CBC parameters except haemoglobin. ${ }^{9}$ Red blood cell agglutination can give the tail seen on the right side of the chart. ${ }^{9}$

We did a blood smear, stained with Romanovski Giemsa, and examined the cell morphology. We paid particular attention to the arrangement of erythrocytes, which form cylindrical coin columns and agglutinated cluster-like RBCs (clumps). Single schizocytes are also observed.

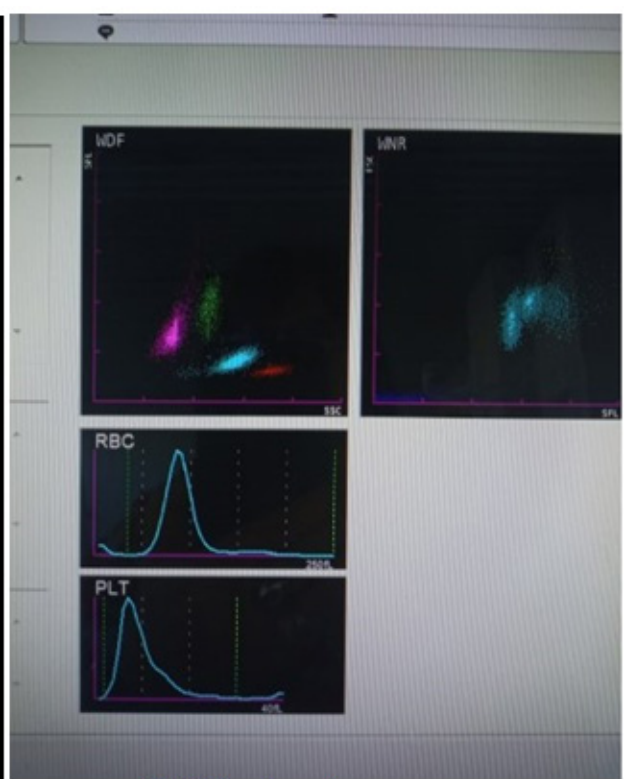

B) After 3 weeks

Figure I Blood picture of the patient with histograms and flags of the Sysmex XNI 000 analyzer.

A spontaneous agglutination is observed in the preparation of blood smear by placing a drop of blood on the glass. Similar agglutination is observed in the EDTA tube at room temperature. The saline test is positive. Due to the abnormal values of $\mathrm{RBC}$ and hematocrit, but the observed dynamic decrease in haemoglobin values within 3-4 days, the biochemical markers for hemolysis were traced (Table 2). 
Table 2 Biochemical markers for hemolysis

\begin{tabular}{lllll}
\hline Biochemistry & & & & \\
\hline Albumin & 37 & $\mathrm{~g} / \mathrm{L}$ & 35 & 53 \\
D. Billirubin & 4.0 & $\mu \mathrm{mol} / \mathrm{L}$ & & 5.13 \\
$\mathrm{LDH}$ & 293 & $\mathrm{U} / \mathrm{L}$ & 90 & 248 \\
Total Protein & 70.0 & $\mathrm{~g} / \mathrm{L}$ & 66 & 83 \\
Toatal Billirubin & 28 & $\mu \mathrm{mol} / \mathrm{L}$ & & 21 \\
Heptoglobin & 1.05 & $\mathrm{~g} / \mathrm{L}$ & 0.5 & 2.2 \\
CRP & 56.3 & $\mathrm{mg} / \mathrm{L}$ & 0 & 5 \\
Free haemoglobin plasma & 0.28 & $\mathrm{~g} / \mathrm{L}$ & & 0.25
\end{tabular}

The slight increase in LDH and total bilirubin at the expense of the indirect fraction is observed. No increased urobilinogen was found in urine. Haptoglobin is within the reference range. It should be borne in mind that it is an acute-phase protein and its levels, in this case, will not be useful for us to reflect the hemolytic process. A cold agglutination titer of 1: 512 was found. Serological virological studies were positive for influenza B, Coxiella burnetii ( $Q$ fever) and in the grey area for EBV.

Three weeks after administration of combination antibiotic therapy (cephalosporins, azithromycin and dexamethasone $(8 \mathrm{mg}$ and decreasing dose), CBC showed normal red blood levels (Table 1 \& Figure 1).

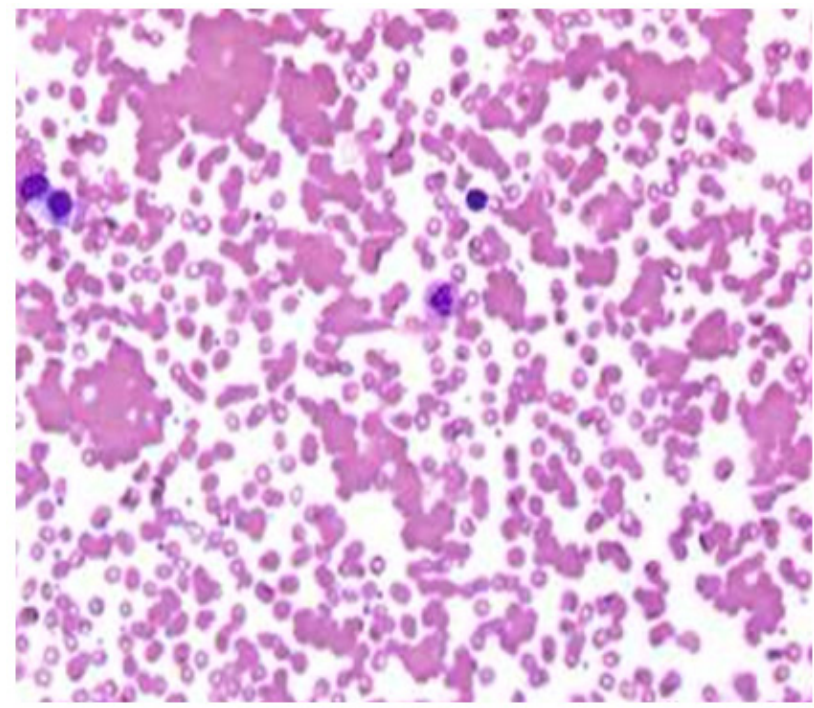

A) Clumps of red blood cells

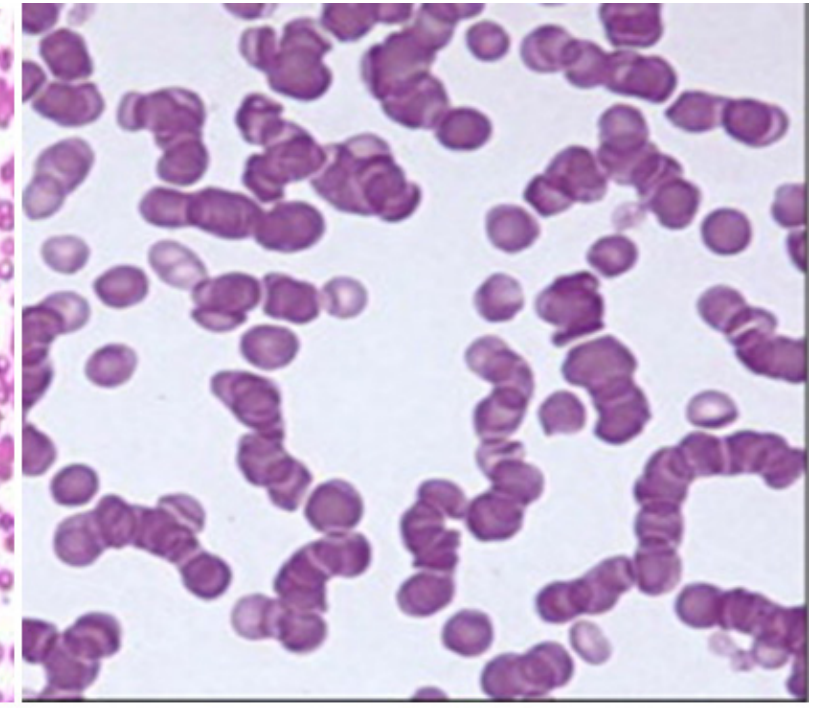

B) Coin Stacking RBC (Rouleaux formation)

Figure 2 Microscopic findings. Peripheral blood smear (K2EDTA) stained by Romanovski-Giemsa and observed changes in red blood cells with agglutination (A) and formation of coin columns (B).

\section{Discussion}

Modern haematology analysers directly measure the parameters $\mathrm{RBC}, \mathrm{Htc}$ and $\mathrm{Hb}$, while $\mathrm{MCV}, \mathrm{MCH}$ and $\mathrm{MCHC}$ are calculated based on them. Automated systems have different channels for counting cells. One channel counts RBCs and platelets, while the other determines leukocytes and haemoglobin levels. In this channel, $\mathrm{RBCs}$ are lysed. In the presence of CA, the number of RBCs may be erroneously reduced and MCVs false, as RBCs micro aggregates are measured as single cells or counted for WBCs. Very large units can even be excluded from the count. Because haemoglobin is determined after lysis of RBC, its value is accurate. All calculated RBC dependent parameters, in this case, will be falsely overestimated, especially $\mathrm{MCHC} .{ }^{4}$ Such constellation is always suspicious of cold agglutinins. High MCHC is a good indicator of sample error (hemolysis, lipemia, erythrocyte agglutination, etc.). ${ }^{10}$ As an approach, in cases of CA, the sample should be delivered within a maximum of 5 min or warmed up before testing. This avoids the temperature influence of the environment.

All CAD patients have hemolysis and show anaemia of varying severity. Hemolysis is immune-mediated by activation of the complement system and in most cases results in intravascular and extravascular lysis of RBC. ${ }^{5}$ In our case, the disease occurred during the winter, in an epidemic flu situation. The etiologic agent, influenza $\mathrm{B}$, leads to secondary CA. The activation of the antibodies is likely to be the result of a lower temperature or a delay in transporting the biological sample to the laboratory. Patients with high titer antibodies may have difficulty not only in automatically counting blood cells but also in serological problems in determining their blood group. ${ }^{11}$

Our patient had no clinical manifestation of hemolytic anaemia. The question arises, whether elevated levels of LDH, bilirubin and free haemoglobin in the plasma can occur artificially in vitro hemolysis during serum and plasma separation. Only a slight increase in bilirubin indicates that we still have in vivo hemolysis.

In the patient, the inflammatory, febrile-intoxication, asthenoadynamic and broncho-pulmonary syndrome and normalization of $\mathrm{CBC}$ were completely controlled within 4 weeks.

\section{Conclusion}

A good knowledge of artificially occurring in vitro phenomena resulting from erythrocyte agglutination can avoid a number of 
misunderstandings or misinterpretations. When observing abnormal histograms, it is imperative to make a blood smear from a fresh Romanovski-Giemsa stained sample. Thus, specific features and changes in the red bloodstream can be visualized microscopically. Informing the clinician will guide him in the correct diagnostic direction.

\section{Funding}

None.

\section{Acknowledgments}

None.

\section{Conflicts of interest}

The author declare have no conflict of interest about the publication of this paper.

\section{References}

1. Zandecki M, Genevieve F, Gerard J, et al. Spurious counts and spurious results on haematology analysers: a review. Part II: white blood cells, red blood cells, haemoglobin, red cell indices and reticulocytes. Int Jnl Lab Hem. 2007;29(1):21-41.

2. Gupta V.Assessment of red blood cell parameters and peripheral smear at different temperatures in case of cold agglutination disease. Ann Med Health Sci Res. 2014;4(Suppl 1):S25-S28.
3. Berentsen S, Beiske K, Tjonnfjord GE. Primary chronic cold agglutinin disease: an update on pathogenesis, clinical features and therapy. Hematology. 2007;12(5):361-370.

4. Topic A, Milevoj Kopcinovic L, Bronic A, et al. Effect of cold agglutinins on red blood cell parameters in a trauma patient: a case report. Biochem Med. 2018;28(3):031001.

5. Nikousefat Z, Javdani M, Hashemnia M, et al. Cold agglutinin disease; a laboratory challenge. Iran Red Crescent Med J. 2015;17(10):e18954.

6. Ercan S, Calıșkan M, Koptur E.70-year old female patient with mismatch between hematocrit and hemoglobin values: the effects of cold agglutinin on complete blood count. Biochem Med (Zagreb). 2014;24(3):391-395.

7. Buttarello M, Plebani M. Automated blood cell counts: state of the art. Am J Clin Pathol. 2008;130(1):104-116.

8. Constantino BT. The red cell histogram and the dimorphic red cell population. LAB Medicine. 2011;42(5):300-308.

9. Constantino BT, Cogionis B. High mean corpuscular hemoglobin concentration: Its causes and effects on automated $\mathrm{CBC}$ results. Can J Med Lab Sci. 2007;69:113-126.

10. Breuer GS, Raveh D, Rudensky B, et al. Remember the blood smear: a clinical laboratory vignette. IMAJ. 2002;4:1089-1090.

11. Javed R, Datta SS, Basu S, et al. Resolution of serologic problems due to cold agglutinins in chronic lymphocytic leukemia. Indian J Hematol Blood Transfus. 2016;32(Suppl 1):290-293. 